\title{
Is there any tip that makes performing EUS-guided drainage easier and safer?
}

Endoscopic ultrasound (EUS)-guided drainage is evolving and no standardized technique for it has been established yet. Availability of dedicated devices and accessories also are lacking. Anderloni et al. described a new technique of stent deployment named Intra Channel Stent Release Technique (ICSRT) in EUS-guided drainage [1], which is summarized as follows: deployment of the proximal part of the stent within the working channel of the echoendoscope and then gentle withdrawal of the scope. As the authors has emphasized, the core of this technique is maintaining stable and secure positioning of the scope during the procedure, thus decreasing the chance of failure.

We totally agree, however, in 2017 we introduced a similar technique called the "modified stent deployment maneuver" [2]. In our previous study about EUSguided hepaticogastrostomy, to stabilize positioning/attachment of the echoendoscope in the high body of the stomach or the cardia during stent placement, we deployed the front half of the dual-flap metal stent, and next the remainder of the stent within the channel of echoendoscope, and then gently pulled out the echoendoscope, leaving the stent. Because stabilization of the scope is most difficult during hepaticogastrostomy, we introduced this technique with a conventional stent deployment system during EUS-guided hepaticogastrostomy.
The concept of our modified stent deployment maneuver and intra-channel stent release technique (ICSRT) is the same, the only difference between the 2 techniques being that ICSRT is performed under complete EUS control using a novel dedicated EUS-guided drainage device (Hot AXIOS ${ }^{\mathrm{TM}}$, Boston Scientific Corp., Malborough, MA, USA). However, fluoroscopic guidance is mandatory during hepaticogastrostomy. Even during EUS-guided choledochoduodenostomy, we recommend doing the procedure under fluoroscopic guidance because it is not possible to anticipate what problems may develop during EUS-guided drainage.

Finally, according to this study, the role of black marker in the catheter for endoscopic deployment of the proximal flange in the AXIOS stent is questionable. Given the high cost of the AXIOS stent with dedicated endoscopic deployment system, a dedicated lumen-apposing stent with conventional deployment system may be more attractive. EUSguided drainage is a challenging procedure and formal training dedicated to EUS-guided drainage is difficult. To develop and establish a standardized protocol for EUS-guided drainage, sharing technical tips and information development of dedicated devices is important.

\section{Competing interests}

None
The authors

\section{Woo Hyun Paik', Do Hyun Park ${ }^{2}$}

1 Department of Internal Medicine and Liver Research Institute, Seoul National University Hospital, Seoul National University College of Medicine, Seoul, Korea

2 Division of Gastroenterology, Department of Internal Medicine, University of Ulsan College of Medicine, Asan Medical Center, Seoul, Korea

\section{Corresponding author}

\author{
Do Hyun Park, MD, PhD, Associate \\ Professor of Internal Medicine \\ University of Ulsan College of Medicine, Asan \\ Medical Center, 88 Olympic-Ro 43-Gil, \\ Songpa-gu, Seoul 05505, Korea \\ Fax: +82-2-3010-8043 \\ dhpark@amc.seoul.kr
}

References

[1] Anderloni A, Attili F, Carrara S et al. Intrachannel stent release technique for fluoroless endoscopic ultrasound-guided lumenapposing metal stent placement: changing the paradigm. Endosc Int Open 2017; 5: E25-E29

[2] Paik WH, Park DH, Choi JH et al. Simplified fistula dilation technique and modified stent deployment maneuver for EUS-guided hepaticogastrostomy. World J Gastroenterol 2014; 20: 5051 - 5059

Bibliography

Dol http://dx.doi.org/10.1055/s-0043-112495 Endoscopy International Open 2017; 05: E985

(c) Georg Thieme Verlag KG

Stuttgart · New York

ISSN 2364-3722 